\title{
Notes on hydrostatic pressure
}

\author{
Gérard C. Nihous ${ }^{1}$
}

Received: 10 June 2015 / Accepted: 10 August 2015 / Published online: 2 September 2015

(C) Springer International Publishing AG 2015

\begin{abstract}
Hydrostatic pressure is one of the most elementary notions encountered in the fields of naval architecture and marine engineering. Its presentation in textbooks is not always clear, however, especially for floating bodies in the presence of waves. This brief article aims to clarify potential elements of confusion about hydrostatic pressure. Atmospheric pressure is duly considered at the air-water interface, and in the presence of waves, a consistent definition is possible that reconciles the points of view of naval architects performing hydrostatic calculations and of hydrodynamicists using, for example, potential theory.
\end{abstract}

Keywords Ship hydrostatics - Ship hydrodynamics ·

Pressure

\section{List of symbols}

\section{Variables}

$f \quad$ Function of space (field)

$F \quad$ Force $(\mathrm{N})$

$g \quad$ Acceleration of gravity $\left(\mathrm{m} \mathrm{s}^{-2}\right)$

$M \quad$ Moment $(\mathrm{N} \mathrm{m})$

$n_{z} \quad$ Vertical component of unit normal vector

$p \quad$ Pressure $(\mathrm{Pa})$

$p_{0} \quad$ Reference pressure, e.g. at air-water interface (Pa)

$p_{\mathrm{d}} \quad$ Dynamic pressure $(\mathrm{Pa})$

$p_{\mathrm{h}} \quad$ Hydrostatic pressure $(\mathrm{Pa})$

$\hat{p}_{\mathrm{d}} \quad$ Alternate definition of dynamic pressure $(\mathrm{Pa})$

Gérard C. Nihous

nihous@hawaii.edu

1 Department of Ocean and Resources Engineering, University of Hawaii, 2540 Dole Street, Honolulu, HI 96822, USA $\hat{p}_{\mathrm{h}} \quad$ Alternate definition of hydrostatic pressure $(\mathrm{Pa})$

$r \quad$ Radial coordinate (m)

$x \quad$ First horizontal coordinate $(\mathrm{m})$

$y \quad$ Second horizontal coordinate $(\mathrm{m})$

$z \quad$ Vertical coordinate measured upward, e.g. from undisturbed air-water interface (m)

\section{Greek letters}

$\begin{array}{ll}\eta & \text { Free surface elevation }(\mathrm{m}) \\ \varphi & \text { Velocity potential }\left(\mathrm{m}^{2} \mathrm{~s}^{-1}\right) \\ \rho & \text { Density }\left(\mathrm{kg} \mathrm{m}^{-3}\right)\end{array}$

\section{Subscripts}

$\begin{array}{ll}\text { A } & \text { Air } \\ \text { w } & \text { Water }\end{array}$

\section{Introduction}

As early as in the third century $\mathrm{BC}$, the great mathematician and physicist Archimedes of Syracuse discovered the fundamental relationship of hydrostatics known since as Archimedes' Principle (for a detailed historical account of Archimedes' contributions to the field of hydrostatics, see, for example, Nowacki and Ferreiro 2011). From simple axiomatic premises and thought experiments, Archimedes was able to establish that the force exerted by a stagnant fluid on an immersed body is vertical upward and equal in magnitude to the weight of the displaced fluid. Intuition certainly supports this important discovery: instead of an immersed body, it suffices to imagine a virtual test volume of fluid in a thought experiment; since the test volume is in equilibrium and that only gravity and fluid forces are assumed to be 
active, the fluid forces exerted on the virtual surface enclosing the test volume must exactly balance the weight of fluid inside. Such an explanation is attractive and essentially correct, but a rigorous proof of Archimedes' Principle in the most general context requires mathematical concepts that would not be available until the advent of advanced calculus, such as Gauss' divergence theorem. Even with the powerful analytical tools now at our disposal, there remain subtleties involved, for example in the treatment of the air-water interface for floating bodies. More details on hydrostatic pressure in a stagnant fluid are provided in Sect. 2.

The situation is much more complex when the fluid is not at rest. In particular, the presence of a surface-wave field requires a hydrodynamic theory to be formulated. Fluid pressure retains a 'hydrostatic' component $p_{\mathrm{h}}$ in this more general context. Physically speaking, though, only total pressure matters and the consideration of 'separate' pressure components is merely a matter of formal convenience. In mathematical terms, the precise definition of $p_{\mathrm{h}}$ in a hydrodynamic framework could include any additive constant that (a) does not depend upon the vertical coordinate and (b) vanishes in the absence of fluid perturbation (waves). The common choice is to set such a constant to zero, i.e. to effectively 'port' the calm-water hydrostatic formula for $p_{\mathrm{h}}$ into the realm of hydrodynamics. By doing so, however, one is left with a function $p_{\mathrm{h}}$ that does not satisfy the air-water boundary condition as before. The consequences of this fact are examined in Sect. 3, where hydrostatic pressure in the presence of a wave field is considered.

\section{Hydrostatic pressure in a stagnant fluid}

The basic case of a stagnant fluid is first considered, where only weight and pressure forces are deemed significant. An $(x, y, z)$ coordinate system is defined where the vertical $z$ axis points upward. Here, the absolute pressure $p$ is by definition hydrostatic, i.e., $p=p_{\mathrm{h}}$. Calling $\rho$ the local fluid density and $g$ the acceleration of gravity, a balance of vertical forces on a fluid element yields the well-known result:

$\frac{\partial p}{\partial z}=-\rho g$

The left-hand-side actually is an exact derivative since $p$ is also found to be independent of the horizontal coordinates. In practical situations when fluid density is uniform, Eq. (1) yields

$p=p_{0}-\rho g z$

At this stage, $p_{0}$ is merely a constant of integration keyed to a choice of origin for the $z$-axis.

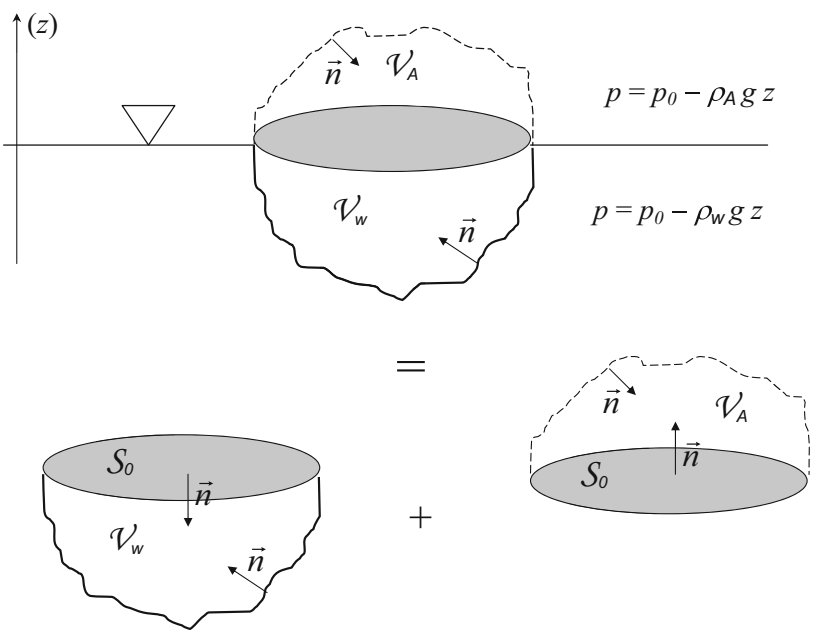

Fig. 1 Schematic diagram for the application of Gauss' divergence theorem to the hydrostatic pressure field surrounding a convex body afloat in a stagnant fluid

For any function of space (field) $f$ defined in a closed volume $V$ bounded by the surface $S$, Gauss' divergence theorem states that

$$
-\iiint_{V} \nabla f \mathrm{~d} V=\iint_{\mathrm{S}} f \vec{n} \mathrm{~d} S
$$

where the minus sign in the left-hand-side indicates that the normal $\vec{n}$ to $S$ points inward, and $\nabla f$ is the gradient of $f$. When $f=p$, the right-hand-side represents the pressure force exerted by the fluid on the body.

Consider a body of volume $\mathcal{V}_{\mathrm{w}}$ completely submerged in water (density $\rho_{\mathrm{w}}$ ). When Eq. (3) is applied in this case with $f=p$, the vertical (non-zero) component of the hydrostatic pressure force $F_{\mathrm{w}}$ is determined and Archimedes' familiar result is obtained: $F_{\mathrm{w}}=\rho_{\mathrm{w}} g \mathcal{V}_{\mathrm{w}}$. For floating bodies, the submerged volume $\mathcal{V}_{\mathrm{w}}$ is bounded by not only the wetted area $\mathcal{S}_{\mathrm{w}}$, as before, but also by the waterplane area $S_{0}$. This suggests a natural choice for the origin of the $z$-axis at the water surface; accordingly, $p_{0}$ is the atmospheric pressure (at 'water level'). Here, the divergence theorem yields

$F_{\mathrm{w}}=\rho_{\mathrm{w}} g \mathcal{V}_{\mathrm{w}}+p_{0} \mathcal{S}_{0}$

With $p_{0}$ about an order of magnitude larger than $\rho_{\mathrm{w}} g$, it seems difficult or arbitrary to simply ignore the term arising from pressure forces that would be exerted on $\mathcal{S}_{0}$ (in general, $\mathcal{S}_{0}$ is not a physical boundary unless the body floats exactly awash with the water surface). To resolve the issue, the dry surface of the body $\mathcal{S}_{\mathrm{A}}$ also may be considered. With little loss of generality, the body can be taken to be convex, with a dry volume $\mathcal{V}_{\mathrm{A}}$ bounded by $\mathcal{S}_{\mathrm{A}}$ and $\mathcal{S}_{0}$. In this case, the total volume is $\mathcal{V}=\mathcal{V}_{\mathrm{w}}+\mathcal{V}_{\mathrm{A}}$ as illustrated in Fig. 1. As long as the density of air $\rho_{\mathrm{A}}$ does not vary much, air pressure can be 
written as $p_{\mathrm{A}}=p_{0}-\rho_{\mathrm{A}} g z$. The divergence theorem can be applied to the dry volume to determine the vertical pressure force $F_{\mathrm{A}}$ exerted by air on the body:

$F_{\mathrm{A}}=\rho_{\mathrm{A}} g \mathcal{V}_{\mathrm{A}}-p_{0} \mathcal{S}_{0}$

It follows that the overall vertical fluid force $F$ is equal to

$$
F=g\left(\rho_{\mathrm{w}} \mathcal{V}_{\mathrm{w}}+\rho_{\mathrm{A}} \mathcal{V}_{\mathrm{A}}\right)
$$

The moment exerted by fluid pressure forces about the origin is

$$
\begin{aligned}
\vec{M} & =\iint_{S} \vec{r} \times p \vec{n} \mathrm{~d} S \\
& =\iint_{\mathcal{S}_{\mathrm{w}}} \vec{r} \times p_{\mathrm{w}} \vec{n} \mathrm{~d} S+\iint_{\mathcal{S}_{\mathrm{A}}} \vec{r} \times p_{\mathrm{A}} \vec{n} \mathrm{~d} S,
\end{aligned}
$$

where $\vec{r}$ is the position vector. After expanding the cross products and expressing the pressure via Eq. (2) in both water and air, it is straightforward to show that, by applying Gauss' divergence theorem, only integrands containing $z n_{z}$ contribute non-zero terms. More specifically, we obtain

$\vec{M}=\rho_{\mathrm{w}} g \iint_{\mathcal{S}_{\mathrm{w}}}\left(\begin{array}{c}-y z \\ x z \\ 0\end{array}\right) n_{z} \mathrm{~d} S+\rho_{\mathrm{A}} g \iint_{\mathcal{S}_{\mathrm{A}}}\left(\begin{array}{c}-y z \\ x z \\ 0\end{array}\right) n_{z} \mathrm{~d} S$

Let $C_{\mathrm{w}}=\left(x_{\mathrm{w}}, y_{\mathrm{w}}, z_{\mathrm{W}}\right)$ and $C_{\mathrm{A}}=\left(x_{\mathrm{A}}, y_{\mathrm{A}}, z_{\mathrm{A}}\right)$ be the volume centroids of $\mathcal{V}_{\mathrm{w}}$ and $\mathcal{V}_{\mathrm{A}}$, respectively. Applying Eq. (3) with $f=y z$ and $f=x z$ leads to the following final form:

$\vec{M}=g\left(\begin{array}{c}\rho_{\mathrm{w}} \mathcal{V}_{\mathrm{w}} y_{\mathrm{w}}+\rho_{\mathrm{A}} \mathcal{V}_{\mathrm{A}} y_{\mathrm{A}} \\ -\rho_{\mathrm{w}} \mathcal{V}_{\mathrm{w}} x_{\mathrm{w}}-\rho_{\mathrm{A}} \mathcal{V}_{\mathrm{A}} x_{\mathrm{A}} \\ 0\end{array}\right)$

Because $\rho_{\mathrm{A}} \ll \rho_{\mathrm{w}}$, by nearly three orders of magnitude, the terms arising from air pressure forces can be neglected in Eqs. (6) and (7) as long as $\mathcal{V}_{\mathrm{A}}$ is not much larger than $\mathcal{V}_{\mathrm{w}}$. Here, the traditional hydrostatic formulas for a floating body are derived without making any assumption on $p_{0}$, but by invoking the fact that air density is much smaller than that of water. As a matter of fact, $p_{0}$ does not explicitly appear in the above results. Also, the case of submerged bodies is recovered if we set $\rho_{\mathrm{A}}=\rho_{\mathrm{w}}$ in Eqs. (6) and (7).

With concave floating bodies, for which $\mathcal{V}=\mathcal{V}_{\mathrm{w}}-\mathcal{V}_{\mathrm{A}}$, a similar approach can be followed. Such geometries are perhaps more representative of ship hulls. Results are given below but details are omitted:

$$
\begin{aligned}
& F=g\left(\rho_{\mathrm{w}} \mathcal{V}_{\mathrm{w}}-\rho_{\mathrm{A}} \mathcal{V}_{\mathrm{A}}\right) \\
& \vec{M}=g\left(\begin{array}{c}
\rho_{\mathrm{w}} \mathcal{V}_{\mathrm{w}} y_{\mathrm{w}}-\rho_{\mathrm{A}} \mathcal{V}_{\mathrm{A}} y_{\mathrm{A}} \\
-\rho_{\mathrm{w}} \mathcal{V}_{\mathrm{w}} x_{\mathrm{w}}+\rho_{\mathrm{A}} \mathcal{V}_{\mathrm{A}} x_{\mathrm{A}} \\
0
\end{array}\right)
\end{aligned}
$$

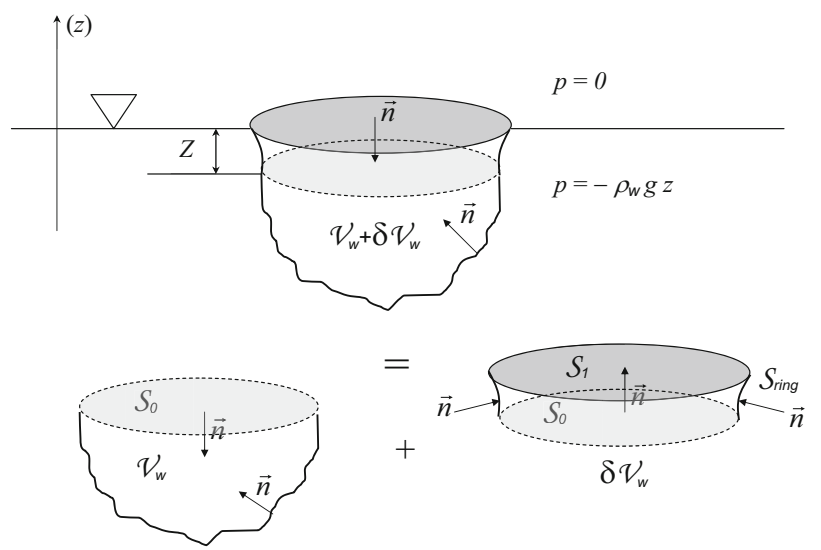

Fig. 2 Schematic diagram for the application of Gauss' divergence theorem to the hydrostatic pressure field surrounding a vertically displaced body afloat in a stagnant fluid

In what follows, $\rho_{\mathrm{A}} \ll \rho_{\mathrm{W}}$ is neglected, and since the overall fluid forces on the body have been shown to be independent of $p_{0}, p_{0}$ can also be set to 0 .

It should be noted that many common textbooks in naval architecture state Archimedes' Principle as a starting point for the study of ship stability, without explicitly expressing hydrostatic pressure and resolving hydrostatic forces applied on submerged or floating bodies (e.g., Gillmer and Jonhson 1982; Lewis 1988; Zubaly 1996; Tupper 2004). Sometimes, $p_{0}$ is implicitly set to zero as in Newman (1977), where atmospheric pressure is formally considered when deriving wave potentials (i.e., Equation (3), p. 239), but is later omitted when dealing with body responses in waves (i.e., Equation (129), p. 289). Other authors recognize that $p_{0}$ also acts on the superstructure of a floating body; they either state (Molin 2002) or demonstrate on a simple shape (Biran 2003) that $p_{0}$ does not result in any net force on the entire body and can, therefore, be set to zero. Semyonov-Tyan-Shansky (1966) stands out for providing an accurate derivation of Eq. (8) in the practical case of thin concave floating bodies similar to ship hulls $\left(\mathcal{V}_{\mathrm{w}}=\mathcal{V}_{\mathrm{A}}\right)$, leading to Equation (4.17), p. 19.

When a floating body is displaced vertically over a distance $Z$ (without disturbing the fluid), the vertical force changes by an amount equal to $\delta F_{\mathrm{w}}=\rho_{\mathrm{w}} g \delta \mathcal{V}_{\mathrm{w}}$, where the incremental (algebraic) submerged volume $\delta \mathcal{V}_{\mathrm{w}}$ is bounded by surfaces $\mathcal{S}_{0}, \mathcal{S}_{1}$ and $\mathcal{S}_{\text {ring }}$, as illustrated in Fig. 2 . Note that if $Z$ is small, the integral of the hydrostatic pressure forces over $S_{\text {ring }}$ is of order $Z^{2}$ and can be neglected; in this case, the only non-zero contribution to the incremental vertical force is equal to $-\rho_{\mathrm{w}} g \mathcal{S}_{0} Z$, a well-known result for the hydrostatic restoring force in the linearized analysis of the motion of floating bodies. While those results are rather obvious, the case of vertically displaced floating bodies in a stagnant fluid was primarily considered to highlight the fact that the effect 
of a wave field on hydrostatic pressure is fundamentally different from the effect of body motion.

\section{Hydrostatic pressure in the presence of a wave field}

We now turn our attention to cases when a wave field is present. With little loss of generality, we focus on floating bodies that are fixed in position, and it is also assumed that the waves under consideration can be described by linear potential theory. The linearized form of Euler's integral provides an expression for the total fluid pressure (where $p_{0}$ has been set to zero):

$p=-\rho_{\mathrm{w}} g z-\rho_{\mathrm{w}} \frac{\partial \varphi}{\partial t}$

Traditionally, the two terms in the right-hand side are defined as the hydrostatic pressure $p_{\mathrm{h}}$ and the dynamic pressure $p_{\mathrm{d}}$ (e.g., Newman 1977). This choice straightforwardly extends the expression $p_{\mathrm{h}}=-\rho_{\mathrm{w}} g z$ that holds in a stagnant fluid. On the free surface $z=\eta$, the pressure is equal to $p_{0}=0$ and this dynamic boundary condition yields a familiar linearized expression for $\eta$ :

$\eta=-\left.\frac{1}{g} \frac{\partial \varphi}{\partial t}\right|_{\eta \approx 0}$

Regarding hydrostatic pressure, an important point of departure from the stagnant fluid case is that it does not satisfy the boundary condition $p_{\mathrm{h}}=0$ on the free surface. Instead, we have $p_{\mathrm{h}}(\eta)=-\rho_{\mathrm{w}} g \eta$, as well as $p_{\mathrm{d}}(\eta)=\rho_{\mathrm{w}} g \eta$. If the hydrostatic pressure force is estimated with Eq. (3) and $f=-\rho_{\mathrm{w}} g z$, as before, Archimedes' familiar theorem does not hold. We obtain an incremental vertical hydrostatic force $\delta F_{\mathrm{w}}$ equal to

$\delta F_{\mathrm{W}}=\rho_{\mathrm{w}} g\left\{\iint_{\mathrm{FS}} \eta n_{z} \mathrm{~d} S+\delta \mathcal{V}_{\mathrm{w}}\right\}$

where FS is the free surface extended inside the body. Possible issues with defining FS are not relevant in the present context; in simpler cases where the Froude-Krylov approximation can be made, there is no ambiguity since the total wave potential is reduced to a function known everywhere (incident wave potential). $\delta \mathcal{V}_{\mathrm{w}}=\iint_{\mathcal{S}_{0}} \eta \mathrm{d} S$ is the incremental (algebraic) submerged volume, as illustrated in Fig. 3. Since the projection of a surface element $\mathrm{d} S$ from FS on $\mathcal{S}_{0}$ is $-n_{z} \mathrm{~d} S$ (with the normal pointing into the fluid), it follows from Eq. (12) that $\delta F_{\mathrm{w}}=0$. With the traditional definition of hydrostatic pressure in the presence of a wave field, the hydrostatic forces exerted on a floating body are independent of this wave field; what this really means is that the dynamic

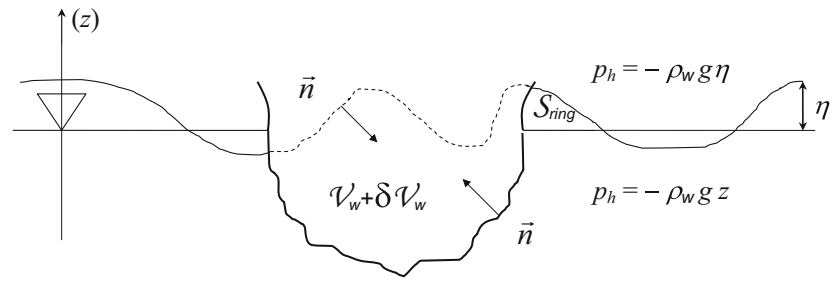

Fig. 3 Schematic diagram for the application of Gauss' divergence theorem to the hydrostatic pressure field surrounding a fixed floating body in the presence of waves

effects of waves are manifest through the dynamic pressure only.

The derivation of this result is not always clear. Newman (1977), for example, attributes it to the smallness of the integral of hydrostatic pressure forces along the thin strip $0<z<\eta$, which is of order $\eta^{2}$ and, therefore, negligible (Section 6.16, page 291). This strip corresponds to $\mathcal{S}_{\text {ring }}$ in Fig. 3. The very same argument, however, would lead to an incorrect result if it were applied to the case of a vertically displaced floating body in a stagnant fluid. It was verified at the end of Sect. 2 that for a small vertical body displacement $Z$, the integral of hydrostatic pressure forces along the thin strip between 0 and $Z$ ( $\mathcal{S}_{\text {ring }}$ in Fig. 2 ) is of order $Z^{2}$ and, therefore, negligible as well. Yet, there is a first-order change in the hydrostatic pressure forces, $\delta F_{\mathrm{w}}=\rho_{\mathrm{w}} g \delta \mathcal{V}_{\mathrm{w}}$. The difference between those two situations is that $p_{\mathrm{h}}$ satisfies different boundary conditions at the water surface. This is why Gauss' divergence theorem leads to different results.

Other respected textbooks in the field of ocean engineering incorrectly treat hydrostatic pressure effects in the presence of a wave field, and such a lapse is more serious than the loose derivation of a correct result. In a generally wellwritten and clear treatise on wave mechanics, McCormick (1973) addresses heaving forces on floating structures in waves (Chapter 4). The hydrostatic restoring force due to the body motion alone, as well as wave-induced effects from the dynamic pressure $p_{\mathrm{d}}$ (under the Froude-Krylov hypothesis), is correctly described. In addition, however, another term is proposed: this "second wave-induced force is the change in the hydrostatic restoring force due to the passage of a wave" and leads to Equation (4.29); in the present notation, this corresponds to an additional vertical force precisely equal to $\rho_{\mathrm{w}} g \delta \mathcal{V}_{\mathrm{w}}=\rho_{\mathrm{w}} g \iint_{\mathcal{S}_{0}} \eta \mathrm{d} S$. Unfortunately, it represents a spurious contribution from the hydrostatic pressure that is at least as large as the Froude-Krylov force determined from the dynamic pressure; the two terms would be equal in the long-wave (shallow draft) limit when the product of the wave number and the body draft tends to zero. Hence, the inclusion of the spurious component amounts to an overestimation of wave forces by, roughly, a factor of two. The same approach can be traced in subsequent publications, e.g. McCormick (2007), e.g. in Equations (4.25, 4.26). 
One could define hydrostatic and dynamic pressure terms $\hat{p}_{\mathrm{h}}$ and $\hat{p}_{\mathrm{d}}$ that would individually satisfy the free surface boundary condition $p=0$. It suffices to write

$$
\left\{\begin{array}{l}
\hat{p}_{\mathrm{h}}=-\rho g(z-\eta) \\
\hat{p}_{\mathrm{d}}=-\rho\left(\frac{\partial \varphi}{\partial t}+g \eta\right)
\end{array}\right.
$$

This would allow Archimedes principle to be valid in the presence of waves, but it would neither simplify the overall analysis nor alter the overall result (i.e., a determination of the total forces exerted on a body). Note that since the wave elevation $\eta$ depends on the horizontal coordinates $x$ and $y$, using the definition of hydrostatic pressure shown in Eq. (13) would yield non-zero hydrostatic horizontal forces.

In closing, we should comment on the fact that naval architects implicitly use the hydrostatic pressure definition $\hat{p}_{\mathrm{h}}$ when estimating wave-induced primary structural loads (e.g., Lewis 1988). The practice consists in estimating sectional buoyancy loads along a ship with the wave profile fixed in time ('frozen'). To consider 'extreme' scenarios, the wavelength is taken to be equal to the ship length, with either a trough (sagging condition) or a crest (hogging condition) midship. Interestingly, the quasi-static loads thus derived are good approximations of the total pressure-induced loads (for a given wave phase and under a Froude-Krylov hypothesis) since the wave under consideration is long enough (or, in relative terms, the draft of the ship shallow enough) to have $\hat{p}_{\mathrm{d}} \approx 0$.

\section{Conclusions}

While it has been recognized that students generally struggle with the concept of hydrostatic pressure (Loverude et al. 2010), this brief article reviewed its application in the context of marine engineering. The basic case of objects immersed in, or floating on a stagnant fluid was first considered. The formulas embodied in Archimedes' principle and used to determine hydrostatic pressure forces and moments can simply be derived with Gauss' divergence theorem. For floating bodies, it was shown that no assumption is needed regarding the pressure $p_{0}$ at the water-air interface (atmospheric pressure). With air regarded as a second fluid, overall fluid forces can be derived where the presence of $p_{0}$ is eliminated; traditional textbook formulas can then be recovered by invoking the relative smallness of air density. Next, the case of a vertically displaced body in a stagnant fluid was addressed to confirm that a change in vertical hydrostatic forces occurs (first-order term for small displacements) even though pressure forces along the additional wetted surface are negligible (second-order). Finally, fixed floating objects in the presence of waves were analyzed, where the total pressure is traditionally split into additive terms, labeled hydrostatic and dynamic, which do not individually satisfy the dynamic free surface condition. As a result, Archimedes' principle does not hold for this (pseudo) hydrostatic field. Instead, it was shown that no change in vertical hydrostatic forces occurs even though passing waves locally modify body submergence. It was found that this point is not well explained in textbooks, or that sometimes, a spurious 'waveinduced hydrostatic' term is even proposed. In the latter case, the error committed would be large, with a relative overestimation of wave-induced forces as large as a factor of two. Finally, definitions of hydrostatic and dynamic pressure terms were proposed such that Archimedes' principle would be applicable to the hydrostatic pressure field, even though this would merely be a matter of formalism (or preference). It was argued that quasi-static ship load (primary strength) calculations performed by naval architects implicitly use the 'alternate' definition of hydrostatic pressure, and that 'hydrostatic' forces thus estimated actually reflect the effect of total pressure.

\section{References}

Biran A (2003) Ship hydrostatics and stability. ButterworthHeinemann/Elsevier, Oxford

Gillmer TC, Jonhson B (1982) Introduction to naval architecture. Naval Institute Press, Annapolis

Lewis EV (1988) Principles of naval architecture (second revision). Stability and strength, vol I. The Society of Naval Architects and Marine Engineers (SNAME), Jersey City

Loverude ME, Heron PRL, Kautz CH (2010) Identifying and addressing student difficulties with hydrostatic pressure. Am J Phys 78:75-85

McCormick ME (1973) Ocean engineering wave mechanics. In: McCormick ME, Bhattacharyya R (eds) A volume in ocean engineering. A Wiley series. Wiley, New York

McCormick ME (2007) Ocean wave energy conversion, 4th edn. Dover Publications Inc., Mineola

Molin B (2002) Hydrodynamique des Structures Offshore. Éditions 362 TECHNIP, Paris, France (in French)

Newman JN (1977) Marine hydrodynamics. MIT Press, Cambridge

Nowacki H, Ferreiro LD (2011) Historical roots of the theory of hydrostatic stability of ships. In: Neves MAS et al (eds) Contemporary ideas on ship stability and capsizing in waves. Fluid mechanics and its applications, vol 96, pp 141-180

Semyonov-Tyan-Shansky V (1966) Statics and dynamics of the ship. Peace Publishers, Moscow

Tupper EC (2004) Introduction to naval architecture, 4th edn. Butterworth-Heinemann/Elsevier, Oxford

Zubaly RB (1996) Applied naval architecture. Cornell Maritime Press, Centreville 